\title{
Mostly worse, occasionally better: impact of COVID-19 pandemic on the mental health of Canadian children and adolescents
}

\author{
Katherine Tombeau Cost ${ }^{1}$ (1) Jennifer Crosbie ${ }^{1,2} \cdot$ Evdokia Anagnostou $^{3,4} \cdot$ Catherine S. Birken $^{3,5} \cdot$ Alice Charach $^{1,2}$. \\ Suneeta Monga ${ }^{1,2} \cdot$ Elizabeth Kelley $^{6} \cdot$ Rob Nicolson $^{7}$. Jonathon L. Maguire ${ }^{3,8} \cdot$ Christie L. Burton ${ }^{1}$. \\ Russell J. Schachar ${ }^{1,2} \cdot$ Paul D. Arnold ${ }^{9,10}$ • Daphne J. Korczak ${ }^{1,2}$
}

Received: 26 October 2020 / Accepted: 9 February 2021 / Published online: 26 February 2021

(c) Springer-Verlag GmbH, DE part of Springer Nature 2021

\begin{abstract}
This large cross-sectional study examined the impact of COVID-19 emergency measures on child/adolescent mental health for children/adolescents with and without pre-existing psychiatric diagnoses. Using adapted measures from the CRISIS questionnaire, parents of children aged 6-18 $(N=1013 ; 56 \%$ male; $62 \%$ pre-existing psychiatric diagnosis) and self-reporting children/adolescents aged 10-18 $(N=385)$ indicated changes in mental health across six domains: depression, anxiety, irritability, attention, hyperactivity, and obsessions/compulsions. Changes in anxiety, irritability, and hyperactivity were calculated for children aged 2-5 years using the Strengths and Difficulties Questionnaire. COVID-19 exposure, compliance with emergency measures, COVID-19 economic concerns, and stress from social isolation were measured with the CRISIS questionnaire. Prevalence of change in mental health status was estimated for each domain; multinomial logistic regression was used to determine variables associated with mental health status change in each domain. Depending on the age group, 67-70\% of children/adolescents experienced deterioration in at least one mental health domain; however, 19-31\% of children/adolescents experienced improvement in at least one domain. Children/adolescents without and with psychiatric diagnoses tended to experience deterioration during the first wave of COVID-19. Rates of deterioration were higher in those with a pre-exiting diagnosis. The rate of deterioration was variable across different age groups and pre-existing psychiatric diagnostic groups: depression 37-56\%, anxiety 31-50\%, irritability 40-66\%, attention 40-56\%, hyperactivity 23-56\%, obsessions/compulsions 13-30\%. Greater stress from social isolation was associated with deterioration in all mental health domains (all ORs 11.12-55.24). The impact of pre-existing psychiatric diagnosis was heterogenous, associated with deterioration in depression, irritability, hyperactivity, obsession/compulsions for some children (ORs 1.96-2.23) but also with improvement in depression, anxiety, and irritability for other children (ORs 2.13-3.12). Economic concerns were associated with improvement in anxiety, attention, and obsessions/compulsions (ORs 3.97-5.57). Children/adolescents with and without pre-existing psychiatric diagnoses reported deterioration. Deterioration was associated with increased stress from social isolation. Enhancing social interactions for children/adolescents will be an important mitigation strategy for current and future COVID-19 waves.
\end{abstract}

Keywords COVID-19 $\cdot$ Child and adolescent psychiatry $\cdot$ Neurodevelopmental disorders

\section{Introduction}

COVID-19 emergency measures, leading to the closure of schools, recreation centres, and organized activities, have resulted in widespread disruption to all aspects of the lives and routines of children and adolescents. COVID-19

Katherine Tombeau Cost

Katherine.cost@sickkids.ca

Extended author information available on the last page of the article emergency measures began in Ontario, Canada on March 12, 2020 and largely remained under "lockdown", including the closure of all schools, nearly all childcare centres, all non-essential retail shops, many local and provincial parks, and in-person medical and dental visits except for emergency services until June 22, 2020. Daily routines afforded by school and extracurricular activities are instrumental in maintaining physical activity, regulating sleep cycles, and providing social interactions, all key protective factors for children's and adolescents' mental health [1-3]. Emerging 
literature from countries experiencing a high prevalence of COVID-19, such as China, suggests significant psychological impact in children and adolescents[4]. Concerns about being infected and living in an area with higher viral prevalence were associated with increased depressive and anxious symptoms among adolescents (44\% and 37\%, respectively) $[4,5]$. Published studies [6-9], however, have provided little to no information about the relative impacts of COVID-19 exposure and implementation of emergency measures, e.g., compliance with directives and stress from social isolation, on child and adolescent mental health. Moreover, exposure to virus and imposed emergency measures may vary differentially across age groups (infancy to 18 years) and previous diagnosis with either mental health or neurodevelopmental disorders or both. Further, while the majority of previous studies have focused on depression and anxiety, this study examined six mental health domains. Several commentaries have highlighted the likely deleterious effects of COVID-19 emergency measures on child and adolescent mental health $[10,11]$, yet it is also possible that some children and adolescents may experience improvements in mental health compared to before the pandemic [12,13] due to reduced school and/or social stress. Understanding factors associated with improvement, as well as deterioration, of mental health is important for understanding resiliency and considering interventions to ameliorate outcomes for those experiencing mental health deterioration.

Our primary objective was to examine the impact of emergency measures on mental health status as compared with pre-pandemic mental health across six domains: depression, anxiety, irritability, attention, hyperactivity, and obsessions/compulsions in children $2-18$ years of age, as reported by parents and in children and adolescents 10-18 years of age, as self-reported. Secondary objectives included examining these impacts, by mental health domain, among children with and without pre-COVID psychiatric and neurodevelopmental diagnoses, and to identify risk and protective factors for changes in mental health.

\section{Materials and methods}

\section{Participants}

This cross-sectional study was embedded within four ongoing study cohorts: two clinically-referred mental health and neurodevelopmental cohorts and two community cohorts: (i) SickKids Psychiatry: children/adolescents ages 6-18 years in the Greater Toronto Area referred to an outpatient mental health clinic for evaluation of mental health concerns including but not limited to depression and anxiety disorders, attention-deficit/hyperactivity disorder (ADHD), obsessive-compulsive disorder (OCD), disruptive behaviour disorders (see Supplement for cohort details); (ii) the Province of Ontario Neurodevelopmental Disorder (POND) network[14]: children/adolescents ages 6-18 years throughout the province of Ontario receiving care at outpatient clinics with neurodevelopmental disorders, including autism spectrum disorders (ASD), ADHD, OCD, and intellectual disability; (iii) The Applied Research Group for Kids (TARGet Kids!)[15]: healthy children recruited from ages birth to 5 years in the Greater Toronto Area, and participating in a primary care practice-based research network; and (iv) Spit for Science [16]: a population-based sample of children/ adolescents ages 6-18 years recruited at an urban science museum. The sample is primarily made up of children and adolescents from the southern Ontario region. These four cohorts were selected as established cohorts with a diverse, existing participant base for this study on the effects of COVID-19 on child health and mental health. These cohorts include children across all age ranges up to 18 years. By inclusion of both clinical and community samples (e.g., children and adolescents with pre-COVID mental health and/ or neurodevelopmental diagnoses), the impacts of the pandemic could be examined across multiple important populations. Parents who had previously consented to be contacted were sent an email about participation with a separate link to send to their child if their child was between the ages of 10 and 18 years and interested in participating. This study was approved at all institutional research ethics boards and all participants provided informed consent/assent.

Parents of children $2-18$ years old $(n=1013 ; 53.2 \%$ response rate) completed online questionnaires using REDcap $[17,18]$. Youth $10-18$ years $(n=347)$ completed selfreport questionnaires though a separate email link to their parents' email address. This sample is a convenience sample and participants were not selected for invitation or inclusion based on any specific characteristics. Emergency measures were implemented in Canada in mid-March 2020; all data collection using online questionnaires were completed from April 15, 2020 to June 19, 2020 (Fig. 1). Some data for use in calculating the mental health status change score (as described below) for children ages 2-5 years old was collected from August 1, 2018 through February 29, 2020. Analysis was completed through July 31, 2020.

\section{Measures}

\section{Child/adolescent mental health status change}

Among children/adolescents 6-18 years, mental health change was assessed separately by domain using a singleitem adapted version of the international CRISIS Questionnaire, which measures impact of the pandemic so that it can be compared across cultures [19,20]. Parents and self-reporting youth were asked, "Compared to the time 
Fig. 1 Flow diagram for participant inclusion in parents of children ages $2-18$ years old and children/youth ages 10-18 years old

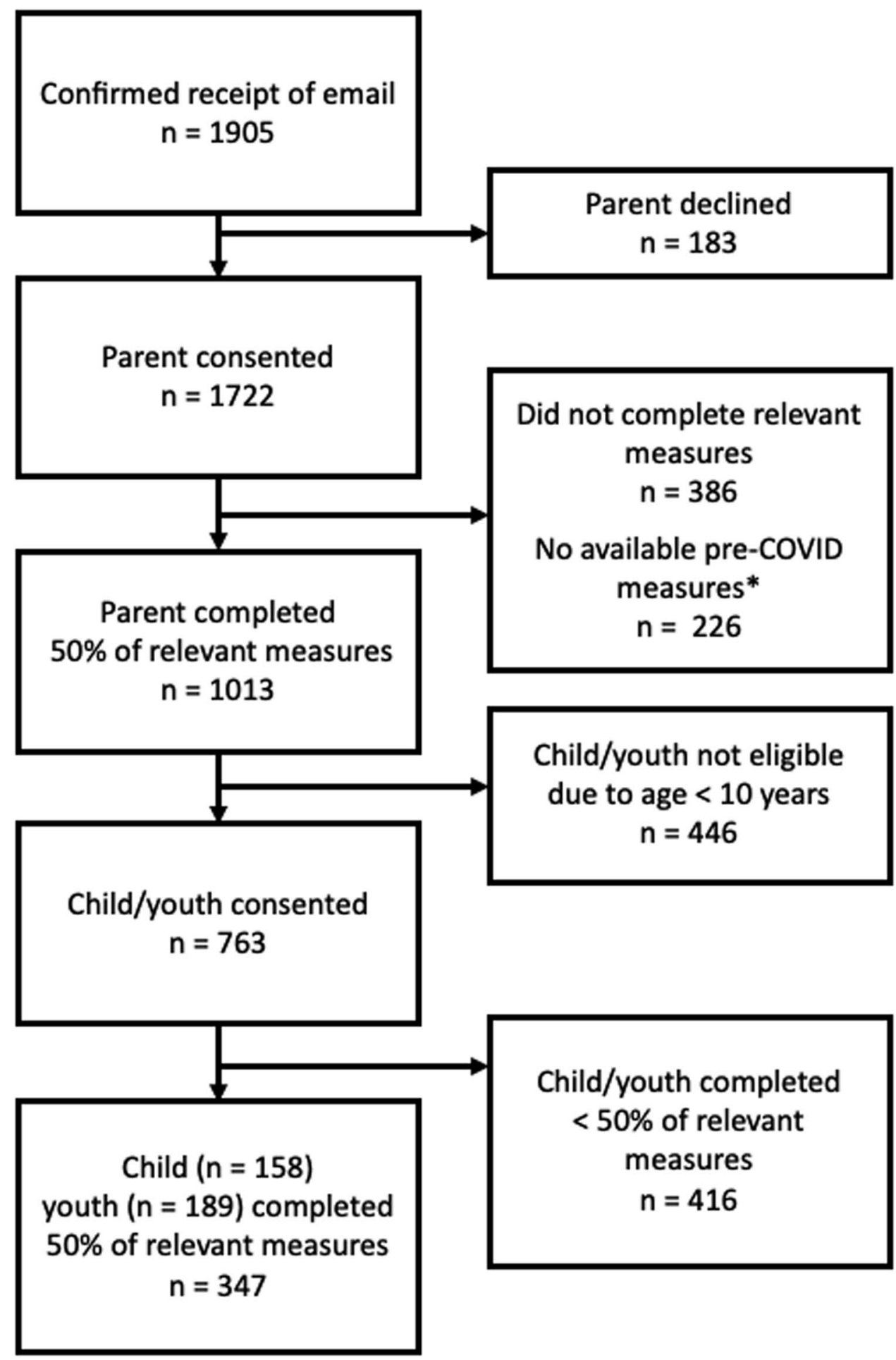

BEFORE the COVID-19 crisis, how is your child's (your) overall mood", using a 5-point Likert scale (1=a lot worse; $5=\mathrm{a}$ lot better). This was repeated for each of the 6 mental health domains (depression, anxiety, irritability, attention, hyperactivity, obsessions/compulsions). Previous research has demonstrated the validity of similar single items measures in assessing psychological distress [21, 22].
Parents of children ages 2-5 years old completed either the Preschool Strengths and Difficulties Questionnaire (preschool SDQ, ages 2-4 years), or the SDQ (for children age 5) $[23,24]$. Three sub-scales of the pre-school SDQ/SDQ that align with the domains assessed in the older children were used: emotional problem subscale for anxiety, conduct problems subscale for irritability, and hyperactivity subscale 
for hyperactivity. The conduct problems subscale items in the pre-school SDQ and SDQ align most closely with the construct of irritability [25], a core symptom of oppositional defiant disorder which is the most common presentation of behaviour problems in the pre-school age group [26].

\section{Demographics}

Parent-reported household income, child age, race/ethnicity, sex, gender identity, and pre-COVID psychiatric diagnoses using items adapted from the CRISIS questionnaire, an instrument designed by an international collaboration to examine mental health during the COVID-19 pandemic [27], and the CRISIS-AFAR adapted for autism and related neurodevelopmental conditions [28] (see Supplemental Table 1 for all individual items and response frequencies).

\section{Economic impact of COVID-19}

Determined using two parent-reported items from the CRISIS questionnaire [27].

\section{Compliance with emergency measures/stay-at-home directives}

Determined using items adapted from the CRISIS questionnaire [27].

\section{COVID-19 exposure}

Determined by responses to two items adapted from the CRISIS questionnaire regarding COVID-19 diagnosis in the parent, child, or family member. An affirmative response to either item was coded as "exposure".

\section{Stress from social isolation}

Assessed using four items from the CRISIS questionnaire [27]. Item responses were provided on a five-point Likert scale $(1=$ not at all; $5=$ extremely) from which the mean score of stress from social isolation was calculated, with higher scores indicating a higher level of stress (see Supplemental Table 1 for all individual items and response frequencies).

\section{Data analysis}

Data were analysed in R [29] and R Studio version 1.2.1335 [30]. We used mice [31] for imputation $(n=10)$ of missing item-level covariate and predictor data where participants had $<50 \%$ missing data on all items required for analysis [32-34]. For scale predictors (economic impact of COVID19 , compliance with stay-at-home directives, stress from social isolation), item responses were averaged and standardised on a scale from 0 to 1 to facilitate comparisons.

\section{Mental health status change}

For responses from parents of children ages 6-18 years, we created 3 categories using the six items on mental health status change by combining scores of 1 and 2 (a lot and a little worse) into a category of "deteriorated"; scores of 3 (about the same) into "unchanged"; 4 and 5 (a little and a lot better) into "improved". Responses by youth report were coded in an identical process. For children 2 to 5 years, a difference score was computed using scores obtained during the study period and data collected on the SDQ in the 18 months prior to COVID-19 from the longitudinal TARGet Kids! Cohort [15]. Improved, deteriorated, and unchanged was operationalized using a change of 2 points as the minimally clinically significant difference in SDQ scores (difference of $\leq-2$ indicated improvement, difference $\geq 2$ indicated deterioration, and difference $<2$ and $>-2$ indicated unchanged) [35].

For child/adolescents 10-18 years, child-parent informant agreement on mental health status change was examined using the $X^{2}$ statistic (Supplement Table 4).

Improvement or deterioration in any domain across all domains for children/adolescents 6 to 18 years old and for children 2-5 years old was examined with prevalence estimates. The prevalence of "improved', "unchanged", or "deteriorated" ratings on the six mental health domains by informant, by child age group, as well as across all participants, and separately in children with and without preCOVID psychiatric diagnoses, were then examined with prevalence estimates. Pre-existing psychiatric diagnoses, assessed with the question, "Has a health or educational professional ever told you that your child had any of the following health conditions? (check all that apply)", from the CRISIS questionnaire, were grouped into four categories: (1) mental health (MH: depression, anxiety, OCD, ADHD, or learning disorder); (2) autism spectrum disorder (ASD: autism spectrum disorder, developmental delay, other neurodevelopmental disorder); (3) comorbid mental health and autism spectrum disorder/developmental delay (MH + ASD: including both listed MH and ASD diagnoses); and (4) no psychiatric diagnosis (NO DX: participants without either $\mathrm{MH}$ or ASD diagnoses).

\section{Risk and protective factors}

Cronbach's alpha examined the internal consistency of scales assessing economic impact, compliance with stay-at-home directives, and stress from social isolation within our sample. For children ages 6-18 years old, multinomial logistic regressions [36] with parent-informant outcome (improved, unchanged, deteriorated) for each of the 6 domains were 
conducted to determine the relative impacts of compliance with emergency measures, stress from social isolation, and COVID exposure, while controlling for child age, sex and ethnicity, household income, COVID-related economic concerns, and any pre-COVID diagnosis.

\section{Results}

\section{Participant characteristics}

The sample included 1013 parents of children/adolescents of 1905 parents who initially confirmed receipt of the study email. Children and adolescents were two to 18 years old [ $2-5$ years, $n=54 ; 6-9$ years, $n=392 ; 10-12$ years, $n=276$; $13-18$ years, $n=291$; mean (SD) age in years $=10.46$ (3.58)]. Overall, 56.1\% $(n=568)$ were male. Of the 763 children and adolescents who consented to participate, 347 completed outcome measures. The mean (SD) age in years of children and adolescents was 13.05 (2.53) and 51.8\% were male. A summary of participant demographic variables is provided in Table 1 and flow diagram in Fig. 1.

\section{Mental health change status}

On examination of the combined, across-domain prevalence of parent-report deterioration in any of the six domains assessed, $70.2 \%$ (651 of 927) of children and adolescents ages 6-18 were faring worse in at least one domain. Among 2 to 5 -year-old children, $66.7 \%$ (36 of 54) were faring worse in at least one of the three domains assessed. For the combined, across-domain prevalence of improvement in any of the six domains, $19.5 \%$ (181 of 927) of children and adolescents ages 6-18 were faring better in at least one domain. Among children ages 2-5, 31.5\% (17 of 54) of children were faring better in at least one of the three domains.

\section{Mental health status change across all age and informant groups}

Parent report was consistent with youth self-report of changes in depression for the categories improved, unchanged, deteriorated $\left(X^{2}(1)=43.91, p<0.001\right)$. In all other domains, cell sizes for "improved" were insufficient for the $X^{2}$ test, however, parent and child/adolescent reports were consistent for the categories of "deteriorated" and "unchanged" (all $p<0.01$; Supplemental Table 4). Full results are available in Table 2 and Fig. 2a. Deterioration in depression, anxiety, irritability, attention, and hyperactivity was reported in as many as $46.5-53.6 \%$ of children and adolescents, depending on age. Conversely, improvements in these domains were noted in as many as $11.0-19.6 \%$ of participants. In the case of obsession/compulsions, deterioration was noted in $19.7-22.6 \%$ of responders, whereas improvements were noted in $3-4 \%$ of children and adolescents. When data were combined across all ages, the domain with the highest rate of deterioration was attention (47.3\%), assessed in children/adolescents ages 6-18 years (see Table 2 for full results).

\section{Mental health status change across all mental health diagnosis groups}

Children/adolescents with NO DX experienced deterioration in depression, irritability, attention, hyperactivity, and obsession/compulsions of $13.0-40.8 \%$, depending on domain. Deterioration in depression, irritability, attention, and hyperactivity was highest in children and adolescents with ASD (56.1-66.7\%) while deterioration in anxiety and obsession/ compulsions was highest among children and adolescents with $\mathrm{MH}+\mathrm{ASD}$ diagnoses. Conversely, improvements in these domains were noted in as many as $0.9-18.8 \%$ of participants depending on diagnostic category and domain. Prevalence of change in mental health status, by domain, in children with and without pre-COVID mental health and neurodevelopmental disorders are presented in Fig. 2b; Supplemental Table 7.

\section{Risk and protective factors}

Internal consistency for parent-report measures for children 6-18 years old on economic impact of COVID-19 $(\alpha=0.77$; adolescent report was a single item) and stress from social isolation (parent-report $\alpha=0.75$; child/adolescent-report $\alpha=0.68$ ) was acceptable. See Supplemental Tables 1-3 for internal consistency of all other scales and item response frequencies.

We hypothesized that stress from social isolation and COVID-19 exposure would both have negative impacts on all domains of child and adolescent mental health. Deterioration in depression was associated with presence of a pre-COVID psychiatric diagnosis $(\mathrm{OR}=2.04,95 \% \mathrm{CI}$ $1.45-2.86, p<0.001)$ and greater stress from social isolation $(\mathrm{OR}=55.24,95 \%$ CI 24.55-124.30, $p<0.001)$. Deterioration in anxiety was associated with greater stress from social isolation $(\mathrm{OR}=54.36,95 \%$ CI 25.03-118.03, $p<0.001)$. Deterioration in irritability was associated with child European/North American ethnicity/ancestry $(\mathrm{OR}=0.58,95 \%$ CI $0.42-0.80, p=0.001$ ), presence of a pre-COVID diagnosis $(\mathrm{OR}=2.08,95 \%$ CI 1.48-2.92, $p<0.001)$, older child age $(\mathrm{OR}=0.93,95 \% \mathrm{CI} 0.88-0.98, p=0.003)$, and stress from social isolation $(\mathrm{OR}=43.25,95 \%$ CI $19.59-95.46$, $p<0.001)$. Deterioration in attention was associated with greater stress from social isolation $(\mathrm{OR}=24.93,95 \% \mathrm{CI}$ $11.76-52.87, p<0.001)$. Deterioration in hyperactivity was associated with being male $(\mathrm{OR}=0.72,95 \%$ CI $0.52-0.99$, 
Table 1 Participant characteristics

\begin{tabular}{|c|c|c|c|}
\hline & $\%(n)$ & Mean (SD) & $N$ \\
\hline \multicolumn{4}{|l|}{ Full sample, parent-report } \\
\hline Self-report household income (> 80,000 CAD/year) & $72.8 \%(663)$ & - & 911 \\
\hline Parent/caregiver relationship to child (biological or adoptive mother) & $90.4 \%(918)$ & - & 1015 \\
\hline Ethnicity/ancestry of the child & & & 995 \\
\hline Caucasian, European, Non-aboriginal North American ethnicity/ancestry origins & $60.6 \%(603)$ & - & \\
\hline $\begin{array}{l}\text { Single ethnicity/ancestry Caribbean, Latin/Central/South American, African, Asian, } \\
\text { Oceania, North American Aboriginal origins }\end{array}$ & $16.3 \%(162)$ & - & \\
\hline Multiple ethnicity/ancestry origins & $23.1 \%(230)$ & - & \\
\hline Assigned sex of the child & & & 1014 \\
\hline Male & $56.0 \%(568)$ & - & \\
\hline Female & $44.0 \%(446)$ & - & \\
\hline Child/adolescent gender identity (ages 6-18 years only) & & & 958 \\
\hline Boy & $56.3 \%(539)$ & - & \\
\hline Girl & $42.7 \%$ (409) & - & \\
\hline Trans boy & $0.3 \%(3)$ & - & \\
\hline Trans girl & $0 \%(0)$ & - & \\
\hline Non-binary or self-described & $0.7 \%(7)$ & - & \\
\hline Any premorbid mental health or ASD diagnosis (ages 6-18 years only) & & & 959 \\
\hline Mental health diagnosis & $45.6 \%(437)$ & - & \\
\hline ASD/neurodevelopmental diagnosis & $4.3 \%(41)$ & - & \\
\hline Comorbid mental health and ASD/neurodevelopmental diagnosis & $12.1 \%(116)$ & - & \\
\hline No mental health, ASD, or neurodevelopmental diagnosis & $38.1 \%(365)$ & - & \\
\hline Child age (in years) & - & $10.46(3.58)$ & 1015 \\
\hline \multicolumn{4}{|l|}{ Parent-report of children ages $2-5$ years old } \\
\hline Economic impact of COVID $19^{\mathrm{a}}$ & - & $0.06(0.17)$ & 55 \\
\hline Emergency measure compliance $^{\mathrm{a}}$ & - & $0.07(0.18)$ & 55 \\
\hline Stress from social isolation ${ }^{\mathrm{a}}$ & & $0.40(0.29)$ & 25 \\
\hline COVID 19 exposure $^{a}$ (positive exposure) & $5.5 \%(3)$ & - & 55 \\
\hline \multicolumn{4}{|l|}{ Parent-report of child/adolescent ages 6 to 18 years old } \\
\hline Economic impact of COVID $19^{a}$ & - & $0.27(0.25)$ & 959 \\
\hline Emergency measure compliance $^{\mathrm{a}}$ & - & $0.39(0.34)$ & 948 \\
\hline Stress from social isolation ${ }^{\mathrm{a}}$ & - & $0.42(0.23)$ & 948 \\
\hline COVID 19 exposure $^{\mathrm{a}}$ (positive exposure) & $4.2 \%(40)$ & - & 959 \\
\hline \multicolumn{4}{|l|}{ Child/adolescent-report, ages $10-18$ years } \\
\hline Assigned sex of the child/adolescent & & & 347 \\
\hline Male & $51.8 \%(180)$ & - & \\
\hline Female & $48.1 \%(167)$ & - & \\
\hline Child/adolescent gender identity (ages $13-18$ years) & & & 182 \\
\hline Boy & $48.4 \%(88)$ & - & \\
\hline Girl & $50.0 \%(91)$ & - & \\
\hline Trans boy & $1.1 \%(2)$ & - & \\
\hline Non-binary or self-described & $0.5 \%(1)$ & - & \\
\hline Child/adolescent age (in years) & - & $13.05(2.53)$ & 347 \\
\hline Emergency measure compliance $^{\mathrm{a}}$ & - & $0.39(0.33)$ & 347 \\
\hline Stress from social isolation ${ }^{\mathrm{a}}$ & - & $0.36(0.22)$ & 347 \\
\hline
\end{tabular}

${ }^{a}$ Characteristics associated with parent-report economic impact of COVID 19, parent-report COVID 19 exposure, parent- and child/adolescentreport of emergency measure compliance, and parent- and child/adolescent-report of stress associated with social isolation are reported separately for the 2-5 year age group, the 6-18 year parent-report age group, and the 10-18 year child/adolescent-report group as the items included in the scales differed in some groups (see Supplement Table 1 for full details on items and response frequencies) 
Table 2 Prevalence of mental health status change by informant and age group

\begin{tabular}{|c|c|c|c|}
\hline & Improved \% $(n)$ & Unchanged \% $(n)$ & Deteriorated \% (n) \\
\hline \multicolumn{4}{|c|}{ Parent-report of children/adolescents ages $2-18$ years old } \\
\hline Depression $^{\mathrm{a}}$ & $11.6 \%(106)$ & $41.5 \%(381)$ & $46.9 \%(430)$ \\
\hline Anxiety/emotional problems ${ }^{\mathrm{b}}$ & $6.2 \%(60)$ & $50.7 \%(493)$ & $43.1 \%(419)$ \\
\hline Irritability/conduct problems ${ }^{\mathrm{b}}$ & $12.0 \%(113)$ & $42.0 \%(394)$ & $45.9 \%(431)$ \\
\hline Attention $^{\mathrm{a}}$ & $7.1 \%(62)$ & $45.6 \%(396)$ & $47.3 \%(411)$ \\
\hline Hyperactivity ${ }^{\mathrm{b}}$ & $5.2 \%(48)$ & $61.3 \%(566)$ & $33.5 \%(309)$ \\
\hline OCD symptoms ${ }^{\mathrm{a}}$ & $4.0 \%(35)$ & $74.9 \%(659)$ & $21.1 \%(186)$ \\
\hline \multicolumn{4}{|c|}{ Parent-report of children ages $2-5$ years old } \\
\hline Emotional problems & $11.1 \%(6)$ & $57.4 \%(31)$ & $31.5 \%(17)$ \\
\hline Conduct problems & $16.7 \%(9)$ & $53.7 \%(29)$ & $29.6 \%(19)$ \\
\hline Hyperactivity & $14.8 \%(8)$ & $50.0 \%(27)$ & $35.2 \%(19)$ \\
\hline \multicolumn{4}{|c|}{ Parent-report of children ages $6-9$ years old } \\
\hline Depression & $8.8 \%(33)$ & $44.2 \%(165)$ & $46.9 \%(175)$ \\
\hline Anxiety & $5.6 \%(21)$ & $47.9 \%(180)$ & $46.5 \%(175)$ \\
\hline Irritability & $4.1 \%(15)$ & $42.3 \%(153)$ & $53.6 \%(194)$ \\
\hline Attention & $9.0 \%(32)$ & $43.1 \%(153)$ & $47.9 \%(170)$ \\
\hline Hyperactivity & $5.4 \%(19)$ & $56.1 \%(199)$ & $38.6 \%(137)$ \\
\hline OCD symptoms & $3.9 \%(14)$ & $76.5 \%(276)$ & $19.7 \%(71)$ \\
\hline \multicolumn{4}{|c|}{ Parent-report of children ages 10-12 years old } \\
\hline Depression & $11.0 \%(29)$ & $41.4 \%(109)$ & $47.5 \%(125)$ \\
\hline Anxiety & $3.8 \%(10)$ & $55.1 \%(146)$ & $41.1 \%(109)$ \\
\hline Irritability & $8.0 \%(20)$ & $41.0 \%(103)$ & $51.0 \%(128)$ \\
\hline Attention & $7.3 \%(18)$ & $46.6 \%(115)$ & $46.2 \%(114)$ \\
\hline Hyperactivity & $5.3 \%(13)$ & $64.8 \%(160)$ & $30.0 \%(74)$ \\
\hline OCD symptoms & $4.0 \%(10)$ & $74.3 \%(185)$ & $21.7 \%(54)$ \\
\hline \multicolumn{4}{|c|}{ Parent-report of adolescent ages $13-18$ years old } \\
\hline Depression & $15.7 \%(44)$ & $38.1 \%(107)$ & $46.3 \%(130)$ \\
\hline Anxiety & $8.3 \%(23)$ & $49.1 \%(136)$ & $42.6 \%(118)$ \\
\hline Irritability & $9.2 \%(25)$ & $46.1 \%(125)$ & $44.6 \%(121)$ \\
\hline Attention & $4.4 \%(12)$ & $47.9 \%(128)$ & $47.6 \%(127)$ \\
\hline Hyperactivity & $2.2 \%(8)$ & $49.0 \%(180)$ & $48.8 \%(179)$ \\
\hline OCD symptoms & $4.1 \%(11)$ & $73.3 \%(198)$ & $22.6 \%(61)$ \\
\hline \multicolumn{4}{|l|}{ Child-report ages $10-12$ years old } \\
\hline Depression & $12.0 \%(19)$ & $52.2 \%(83)$ & $35.4 \%(56)$ \\
\hline Anxiety & $13.3 \%(21)$ & $46.8 \%(74)$ & $39.9 \%(63)$ \\
\hline Irritability & $11.4 \%(18)$ & $43.7 \%(69)$ & $44.9 \%(71)$ \\
\hline Attention & $8.9 \%(14)$ & $45.6 \%(72)$ & $45.6 \%(72)$ \\
\hline Hyperactivity & $10.1 \%(16)$ & $58.2 \%(92)$ & $31.6 \%(50)$ \\
\hline \multicolumn{4}{|c|}{ Adolescent-report ages $13-18$ years old } \\
\hline Depression & $19.6 \%(37)$ & $33.9 \%(64)$ & $46.6 \%(88)$ \\
\hline Anxiety & $14.1 \%(26)$ & $48.1 \%(89)$ & $37.8 \%(70)$ \\
\hline Irritability & $10.9 \%(20)$ & $40.8 \%(75)$ & $48.4 \%(89)$ \\
\hline Attention & $11.0 \%(20)$ & $35.7 \%(65)$ & $53.3 \%(97)$ \\
\hline Hyperactivity & $8.2 \%(15)$ & $58.8 \%(107)$ & $33.0 \%(60)$ \\
\hline
\end{tabular}

In all ages prevalence analysis, adomains reflect only children/adolescents 6-18 years old and ${ }^{\mathrm{b}}$ domains reflect children/adolescents $2-18$ years old $p=0.043$ ), having a pre-COVID psychiatric diagnosis $(\mathrm{OR}=2.23,95 \%$ CI $1.56-3.19, p<0.001)$, older child age $(\mathrm{OR}=0.92,95 \%$ CI $0.88-0.97, p=0.002)$, and greater stress from social isolation $(\mathrm{OR}=16.74,95 \%$ CI 7.94-35.29, $p<0.001)$. Deterioration in obsession/compulsions was associated with having a pre-COVID psychiatric diagnosis 
A

Prevalence of Mental Health Status Change by Domain by Age Group and Informant

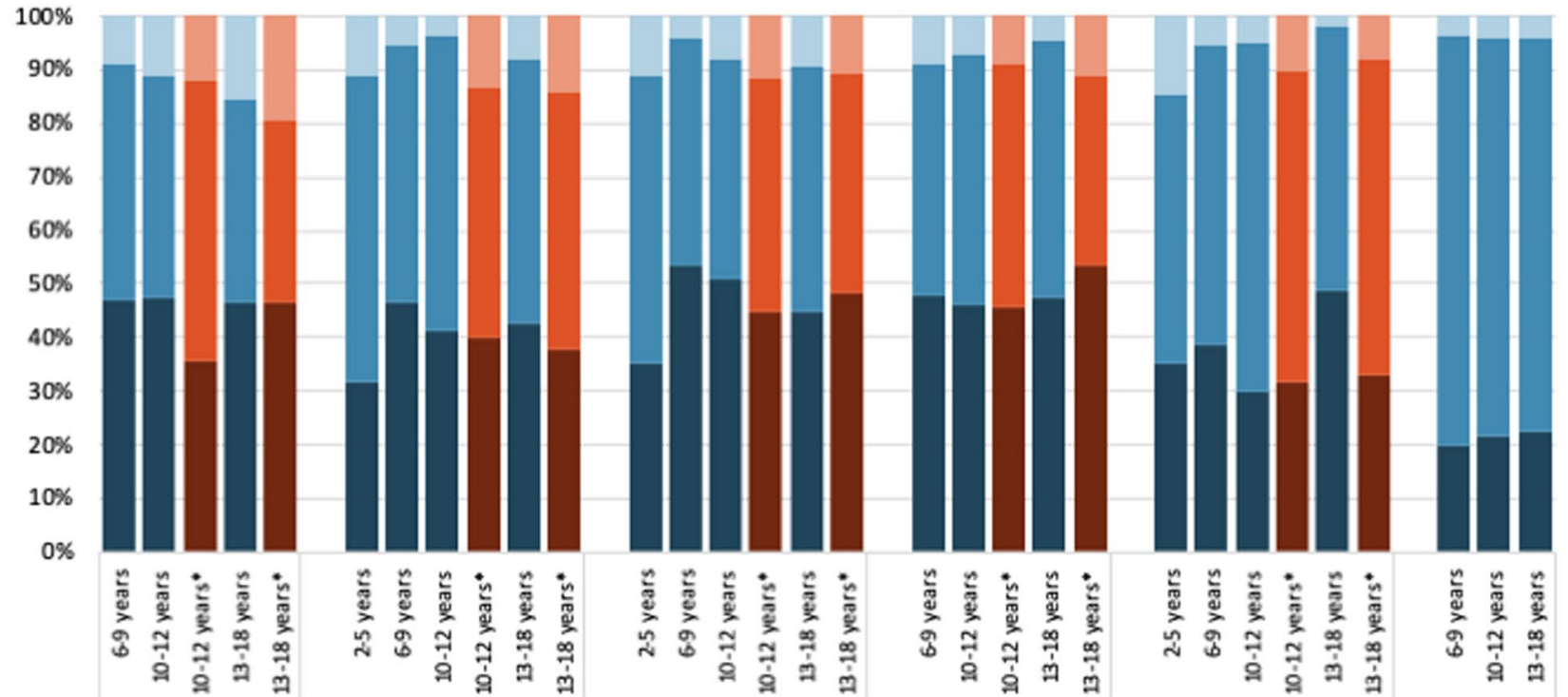

Depression

Anxiety

Irritability

Attention

Hyperactivity

Obsessions/

Compulsions

$\begin{array}{ll}\text { Parent-report } & \text { Child/adolescent-report } \\ \text { = better } & \text { n better } \\ \text { = same } & \text { n same } \\ \text { n worse } & \text { n worse }\end{array}$

B

Prevalence of Mental Health Status Change by Domain by Diagnostic Group

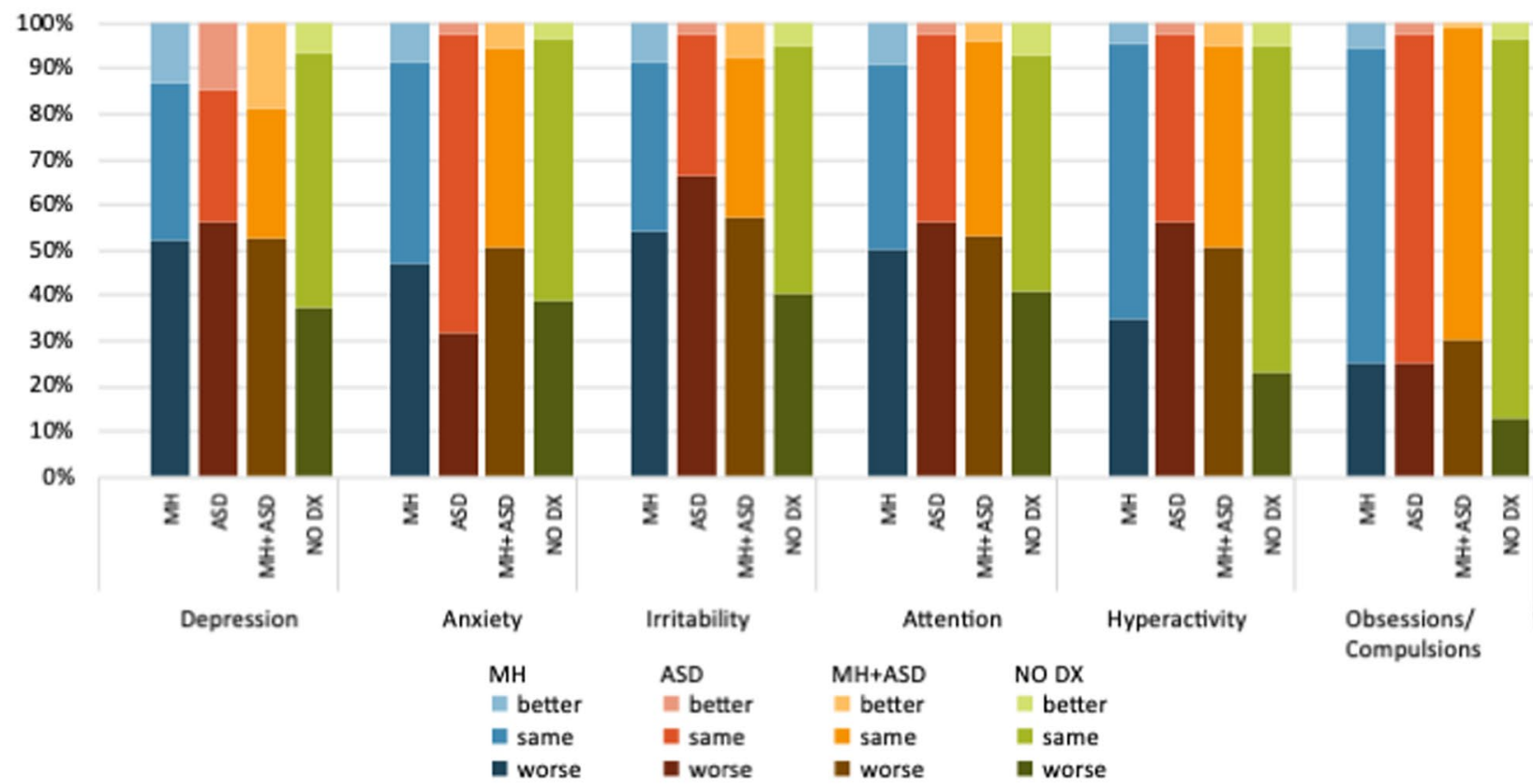

Fig. 2 a Prevalence of mental health status change in 2-18 year-old children/adolescents by domain by age group and informant, b prevalence of mental health status change by domain by diagnostic group 
$(\mathrm{OR}=1.96,95 \%$ CI 1.30-2.98, $p=0.002)$, greater economic concerns $(\mathrm{OR}=2.91,95 \% \mathrm{CI} 1.45-5.85, p=0.003)$, and greater stress from social isolation $(\mathrm{OR}=11.12,95 \% \mathrm{CI}$ 5.01-24.70, $p<0.001$; Table 3).

Improvement in depression was associated with having a pre-COVID psychiatric diagnosis $(\mathrm{OR}=3.12,95 \% \mathrm{CI}$ $1.81-5.37, p<0.001)$ and less stress from social isolation $(\mathrm{OR}=0.16,95 \%$ CI 0.04-0.56, $p=0.004)$. Improvement in anxiety was associated with being female (OR $=1.84,95 \%$ CI $1.02-3.31, p=0.041)$, presence of a pre-COVID psychiatric diagnosis $(\mathrm{OR}=2.42,95 \% \mathrm{CI} 1.17-5.01, p=0.018)$, greater economic concerns $(\mathrm{OR}=5.57,95 \%$ CI $1.64-18.97$, $p=0.006)$, and lower stress from social isolation ( $\mathrm{OR}=0.12$, 95\% CI 0.02-0.62, $p=0.011)$. Improvement in irritability was associated with having a pre-COVID psychiatric diagnosis $(\mathrm{OR}=2.13,95 \%$ CI $1.10-4.13, p=0.024)$. Improvement in attention was associated with greater economic concerns $(\mathrm{OR}=3.97,95 \% \mathrm{CI} 1.29-12.22, p=0.016)$. The model for improvement in hyperactivity was not significant $(p=0.07)$, therefore no further predictors were indicated. Improvement in obsession/compulsions was associated with non-European/North American ethnicity/ancestry $(\mathrm{OR}=2.49,95 \%$ CI 1.18-5.24, $p=0.016)$ and greater economic concerns $(\mathrm{OR}=5.20,95 \%$ CI 1.27-21.21, $p=0.022$; Table 3).

\section{Discussion}

This study, conducted two to four months following implementation of the COVID-19 emergency measures, indicates that many children and adolescents in Canada experienced deterioration across several mental health domains during this time period. More than two thirds of children and adolescents experienced deterioration in mental health, with such deterioration strongly associated with stress related to social isolation. These impacts occurred in the absence of significant COVID-19 disease exposure or economic concerns among study participants, when viral prevalence in the community was estimated at 128.6 cases per 100,000 [37], and prior to the relaxation of lockdown measures.

A significant proportion of children and adolescents experienced deterioration of their mental health, regardless of age, with $70.2 \%$ of children $6-18$ years old and $66.1 \%$ of children 2-5 years old experiencing deterioration in at least one domain. Deterioration in depression was highest among 10-12 year-old children while deterioration in anxiety and irritability was highest among 6- to 9-year-old children. Deterioration in attention, hyperactivity, and obsession/ compulsions were highest among adolescents 13-18 years old. Pre-school-aged children were less likely to show deterioration in anxiety, irritability and hyperactivity, with the lowest rates of deterioration and often the highest rates of improvement in any age group. This indicates that the mental health impacts of the pandemic are greater for schoolaged children, and potentially, in keeping with previous research, related to the loss of daily routines for children of all ages [1].

Notably, children and adolescents experienced relatively similar overall mental health impacts despite their mental health history, though some variations were observed. Children and adolescents with ASD diagnoses were reported to have the greatest deterioration in depression, irritability, attention, and hyperactivity. This may be due to several factors, including the termination of school-based services (including therapeutic services) for children with ASD, in conjunction with challenges associated with online learning, the closure of respite care services, and disruptions to daily routines [38, 39]. More concerning, however, was the significant proportion of children and adolescents with no known pre-COVID mental health or neurodevelopmental challenges who experienced deterioration in a number of mental health domains, with $37-41 \%$ experiencing worsening depression, anxiety, irritability, and attention during the emergency measures.

In comparison, Zhou et al. (2020) reported that $43.7 \%$ and $37.4 \%$, of 12-18 year-old children reported clinically significant rates of depression and anxiety, respectively, in Hubei province, China, which experienced high COVID burden. In the United States, despite well-known challenges with COVID-19, parent-reported behavioural health in children under the age of 18 was only reported to be worse during the pandemic in $14.3 \%$ of the sample [6]. Our findings are concordant with a representative study in Germany [8] that found that compliance with emergency measures and social isolation are significant challenges for children and adolescents. However, we found a higher rate of deterioration in mental health domains, with $17.8 \%$ parent-reported mental health problems in Germany compared to around $70 \%$ with parent-reported mental health deterioration in at least one of the six domains assessed in this study. The much higher rate of mental health deterioration in Canadian children and adolescents may be due to our interest in assessing multiple domains of mental health, any one of which may have been impacted in an individual, as reflected in the relatively lower rates of deterioration within each individual domain (19.7-53.6\%, depending on domain and informant). Our findings extend previous studies in Brazil, China, Germany, India, Italy, Spain, and the United States [4-9, 40-43] by reporting on the mental health status change compared with pre-COVID mental health over several mental health domains, and suggest that the widespread impact of the pandemic on children and adolescents is more likely to be attributable to factors other than the magnitude of viral prevalence and COVID-19 disease burden in the community. 
Table 3 Multinomial logistic regressions with emergency measure compliance, stress from social isolation, COVID exposure, while controlling for self-report household income, economic impact of COVID, child age, child race/ethnicity, and child sex assigned at birth, on Parent Report mental health status change

\begin{tabular}{|c|c|c|c|c|c|c|c|c|}
\hline & \multicolumn{4}{|c|}{$\begin{array}{l}\text { Parent-report of children/adolescent ages } 6-18 \text { years } \\
\text { old: improved vs unchanged }\end{array}$} & \multicolumn{4}{|c|}{$\begin{array}{l}\text { Parent-report of children/adolescent ages } \\
6-18 \text { years old: deteriorated vs unchanged }\end{array}$} \\
\hline & OR & $95 \%$ LLCI & $95 \%$ ULCI & $p$ & OR & 95\% LLCI & $95 \%$ ULCI & $p$ \\
\hline \multicolumn{9}{|l|}{ Depression } \\
\hline Intercept & 0.13 & 0.04 & 0.45 & 0.001 & 0.09 & 0.04 & 0.21 & $<0.001$ \\
\hline Household income & 1.31 & 0.75 & 2.29 & 0.344 & 1.37 & 0.93 & 2.03 & 0.112 \\
\hline Ethnicity & 0.61 & 0.37 & 1.01 & 0.053 & 0.80 & 0.58 & 1.10 & 0.161 \\
\hline Assigned sex & 0.83 & 0.52 & 1.32 & 0.436 & 1.21 & 0.89 & 1.65 & 0.216 \\
\hline Premorbid psychiatric diagnosis & 3.12 & 1.81 & 5.37 & $<0.001$ & 2.04 & 1.45 & 2.86 & $<0.001$ \\
\hline Age & 1.05 & 0.98 & 1.13 & 0.166 & 1.01 & 0.96 & 1.06 & 0.651 \\
\hline COVID exposure & 0.91 & 0.28 & 2.93 & 0.874 & 1.06 & 0.50 & 2.24 & 0.875 \\
\hline COVID economic concerns & 1.71 & 0.63 & 4.61 & 0.290 & 0.56 & 0.28 & 1.10 & 0.093 \\
\hline Emergency measure compliance & 1.55 & 0.81 & 2.99 & 0.188 & 1.09 & 0.69 & 1.72 & 0.706 \\
\hline Stress from social isolation & 0.16 & 0.04 & 0.56 & 0.004 & 55.24 & 24.55 & 124.30 & $<0.001$ \\
\hline \multicolumn{9}{|l|}{ Anxiety } \\
\hline Intercept & 0.05 & 0.01 & 0.24 & $<0.001$ & 0.08 & 0.03 & 0.18 & $<0.001$ \\
\hline Household income & 0.85 & 0.42 & 1.73 & 0.649 & 1.34 & 0.91 & 1.97 & 0.140 \\
\hline Ethnicity & 0.59 & 0.30 & 1.16 & 0.128 & 0.97 & 0.71 & 1.33 & 0.872 \\
\hline Assigned sex & 1.84 & 1.02 & 3.31 & 0.041 & 1.19 & 0.88 & 1.60 & 0.262 \\
\hline Premorbid psychiatric diagnosis & 2.42 & 1.17 & 5.01 & 0.018 & 1.24 & 0.89 & 1.73 & 0.194 \\
\hline Age & 1.00 & 0.91 & 1.09 & 0.947 & 1.01 & 0.96 & 1.06 & 0.724 \\
\hline COVID exposure & 0.45 & 0.06 & 3.50 & 0.442 & 1.27 & 0.62 & 2.62 & 0.510 \\
\hline COVID economic concerns & 5.57 & 1.64 & 18.97 & 0.006 & 1.17 & 0.61 & 2.24 & 0.629 \\
\hline Emergency measure compliance & 0.89 & 0.37 & 2.13 & 0.792 & 0.88 & 0.57 & 1.37 & 0.581 \\
\hline Stress from social isolation & 0.12 & 0.02 & 0.62 & 0.011 & 54.36 & 25.03 & 118.03 & $<0.001$ \\
\hline \multicolumn{9}{|l|}{ Irritability } \\
\hline Intercept & 0.06 & 0.01 & 0.27 & $<0.001$ & 0.24 & 0.11 & 0.57 & 0.001 \\
\hline Household income & 0.69 & 0.37 & 1.29 & 0.243 & 1.21 & 0.84 & 1.75 & 0.311 \\
\hline Ethnicity & 0.76 & 0.41 & 1.39 & 0.365 & 0.58 & 0.42 & 0.80 & 0.001 \\
\hline Assigned sex & 1.20 & 0.68 & 2.11 & 0.529 & 1.31 & 0.96 & 1.79 & 0.086 \\
\hline Premorbid psychiatric diagnosis & 2.13 & 1.10 & 4.13 & 0.024 & 2.08 & 1.48 & 2.92 & $<0.001$ \\
\hline Age & 1.02 & 0.93 & 1.11 & 0.668 & 0.93 & 0.88 & 0.98 & 0.003 \\
\hline COVID exposure & 1.59 & 0.49 & 5.12 & 0.436 & 1.04 & 0.48 & 2.24 & 0.920 \\
\hline COVID economic concerns & 2.81 & 0.88 & 8.96 & 0.081 & 1.18 & 0.60 & 2.32 & 0.623 \\
\hline Emergency measure compliance & 1.20 & 0.52 & 2.73 & 0.670 & 0.95 & 0.61 & 1.48 & 0.816 \\
\hline Stress from social isolation & 0.97 & 0.24 & 3.98 & 0.969 & 43.25 & 19.59 & 95.46 & $<0.001$ \\
\hline \multicolumn{9}{|l|}{ Attention } \\
\hline Intercept & 0.19 & 0.04 & 0.88 & 0.034 & 0.21 & 0.09 & 0.47 & $<0.001$ \\
\hline Household income & 0.70 & 0.35 & 1.40 & 0.315 & 1.09 & 0.75 & 1.59 & 0.657 \\
\hline Ethnicity & 0.80 & 0.44 & 1.45 & 0.461 & 0.83 & 0.61 & 1.14 & 0.250 \\
\hline Assigned sex & 1.19 & 0.69 & 2.08 & 0.529 & 1.15 & 0.85 & 1.55 & 0.367 \\
\hline Premorbid psychiatric diagnosis & 1.48 & 0.80 & 2.73 & 0.210 & 1.38 & 0.99 & 1.92 & 0.059 \\
\hline Age & 0.91 & 0.84 & 1.00 & 0.057 & 0.99 & 0.94 & 1.04 & 0.619 \\
\hline COVID exposure & 1.47 & 0.39 & 5.63 & 0.570 & 1.73 & 0.80 & 3.77 & 0.166 \\
\hline COVID economic concerns & 3.97 & 1.29 & 12.22 & 0.016 & 1.21 & 0.63 & 2.32 & 0.574 \\
\hline Emergency measure compliance & 1.56 & 0.71 & 3.41 & 0.267 & 0.83 & 0.54 & 1.29 & 0.418 \\
\hline Stress from social isolation & 0.93 & 0.24 & 3.68 & 0.918 & 24.93 & 11.76 & 52.87 & $<0.001$ \\
\hline \multicolumn{9}{|l|}{ Hyperactivity } \\
\hline Intercept & 0.19 & 0.03 & 1.14 & 0.070 & 0.26 & 0.11 & 0.62 & 0.002 \\
\hline
\end{tabular}


Table 3 (continued)

\begin{tabular}{|c|c|c|c|c|c|c|c|c|}
\hline & \multicolumn{4}{|c|}{$\begin{array}{l}\text { Parent-report of children/adolescent ages } 6-18 \text { years } \\
\text { old: improved vs unchanged }\end{array}$} & \multicolumn{4}{|c|}{$\begin{array}{l}\text { Parent-report of children/adolescent ages } \\
6-18 \text { years old: deteriorated vs unchanged }\end{array}$} \\
\hline & OR & $95 \%$ LLCI & $95 \%$ ULCI & $p$ & OR & 95\% LLCI & $95 \%$ ULCI & $p$ \\
\hline Household income & 0.60 & 0.28 & 1.27 & 0.180 & 1.16 & 0.77 & 1.75 & 0.470 \\
\hline Ethnicity & 0.71 & 0.34 & 1.47 & 0.358 & 0.98 & 0.70 & 1.36 & 0.888 \\
\hline Assigned sex & 1.27 & 0.66 & 2.45 & 0.468 & 0.72 & 0.52 & 0.99 & 0.043 \\
\hline Premorbid psychiatric diagnosis & 1.39 & 0.67 & 2.86 & 0.375 & 2.23 & 1.56 & 3.19 & $<0.001$ \\
\hline Age & 0.89 & 0.79 & 0.99 & 0.034 & 0.92 & 0.88 & 0.97 & 0.002 \\
\hline COVID exposure & 0.57 & 0.07 & 4.50 & 0.591 & 1.33 & 0.65 & 2.75 & 0.437 \\
\hline COVID economic concerns & 2.51 & 0.64 & 9.88 & 0.188 & 1.81 & 0.94 & 3.48 & 0.075 \\
\hline Emergency measure compliance & 1.37 & 0.53 & 3.50 & 0.516 & 0.95 & 0.60 & 1.51 & 0.833 \\
\hline Stress from social isolation & 0.76 & 0.15 & 3.78 & 0.732 & 16.74 & 7.94 & 35.29 & $<0.001$ \\
\hline \multicolumn{9}{|l|}{ Obsessions/compulsions } \\
\hline Intercept & 0.02 & 0.00 & 0.11 & $<0.001$ & 0.04 & 0.01 & 0.10 & $<0.001$ \\
\hline Household income & 0.91 & 0.38 & 2.16 & 0.823 & 1.08 & 0.70 & 1.64 & 0.732 \\
\hline Ethnicity & 2.49 & 1.18 & 5.24 & 0.016 & 1.15 & 0.80 & 1.66 & 0.456 \\
\hline Assigned sex & 1.35 & 0.67 & 2.73 & 0.397 & 0.97 & 0.68 & 1.38 & 0.877 \\
\hline Premorbid psychiatric diagnosis & 1.47 & 0.66 & 3.25 & 0.347 & 1.96 & 1.30 & 2.98 & 0.002 \\
\hline Age & 1.01 & 0.91 & 1.13 & 0.792 & 1.02 & 0.96 & 1.08 & 0.496 \\
\hline COVID exposure & 0.57 & 0.07 & 4.43 & 0.587 & 0.64 & 0.27 & 1.51 & 0.307 \\
\hline COVID economic concerns & 5.20 & 1.27 & 21.21 & 0.022 & 2.91 & 1.45 & 5.85 & 0.003 \\
\hline Emergency measure compliance & 0.97 & 0.35 & 2.73 & 0.961 & 0.66 & 0.39 & 1.13 & 0.130 \\
\hline Stress from social isolation & 0.33 & 0.06 & 1.79 & 0.197 & 11.12 & 5.01 & 24.70 & $<0.001$ \\
\hline
\end{tabular}

Unchanged was the reference group both for improvement and for deterioration

Values in bolditalic indicate $p<0.05$

Values in bold indicate $p<0.001$

Across all six domains, greater stress from social isolation, including both the cancellation of important events (e.g., graduation ceremonies, school trips, vacations) and the loss of in-person social interactions, was strongly associated with deterioration in mental health across all domains. Less stress from social isolation was associated with improved depression and anxiety. This finding underscores the importance of school, recreation, social activities, and milestone events. An important mitigation strategy to decrease deterioration in all six domains of mental health analysed would be to increase access to recreational and social interactions for children and adolescents of all ages. While following local public health guidelines, children should be supported in attending in-person school, participating in sports and activities, meeting socially with peers, engaging in positive social interactions within the family, and connecting with family and friends through video-chat platforms.

Pre-COVID psychiatric and neurodevelopmental diagnoses were associated with both improvements (anxiety, depression, irritability) and deterioration (hyperactivity, anxiety, obsession/compulsions, depression, irritability) in mental health across domains, thus highlighting the heterogeneity of child and adolescent responses to social and academic expectations across home and school environments. For some children with pre-COVID diagnoses (e.g., those with social anxiety or learning disorders), the stay-athome directives may have provided relief from sources of stress or anxiety, thereby improving their symptoms of anxiety or irritability [13, 44]. For children with other diagnoses (e.g., ASD or ADHD), the loss of structure, consistency, and familiar social and teacher-student interactions may have led to increased irritability and low mood, potentially exacerbated by other factors such as parent stress secondary to the additional tasks of supporting children with schooling [43, 45]. Taken together, these findings on inter-individual differences suggest that children with pre-existing mental health problems are not homogeneous with respect to their response to the COVID pandemic and may experience improvements and deteriorations differentially based on specific symptom profiles within an individual, rather than diagnoses. Further, study results underscore the need for research on effective interventions to mitigate symptom escalation to prevent further distress during the return to school for some children, as well as during subsequent pandemic waves. Mitigation strategies for children with pre-existing psychiatric illness, as well as new onset mental health problems, may include 
ensuring continuous access to outpatient mental health services, as well as access to acute mental health care (e.g., urgent/walk-in mental health clinics, telephone/virtual hotlines) for those in crisis.

Greater COVID economic concerns were associated with improved anxiety, attention, and obsession/compulsions and were also associated with deterioration in obsession/compulsions, indicating that there may be a non-linear relationship between COVID economic concerns and child mental health. COVID economic concerns may be serving as a protective factor in certain situations, including: reduction in work-life-conflict, reduction in stress from employment involving a high risk of COVID-19 exposure, the receipt of emergency financial benefits which may have exceeded wages from low-paying employment, emergency financial benefits alleviating financial constraints concomitant with job loss, or the relative availability of unemployed versus employed parents during the emergency measures. For example, parents who lost employment may have felt less stressed by work-life conflict and/or may have had more time to spend with their children, providing emotional support or assisting with online school, with the knowledge that immediate financial threats were being alleviated by emergency financial benefits. In support of this supposition, a recent systematic scoping review demonstrated that interventions akin to universal basic income [46], much like the emergency financial benefits offered in Canada during the first wave of the pandemic, had strong positive effects on mental health for recipients [47]. Further analyses, both quantitative and qualitative, are required to determine the specific sample characteristics, conditions, and possible mechanisms by which greater economic concerns during COVID-19 were associated with improvement in anxiety, attention, and obsession/compulsions in children and adolescents.

\section{Strengths and limitations}

This study has several strengths, including the survey timing near the peak of the first wave in Canada; the large, diverse sample of children of all ages, with and without mental health and neurodevelopmental disorders; participation of multiple informants; and the incorporation of multiple domains of mental health. This study also has several limitations, including different metrics of mental health status change based on child age; use of a single-item measure of mental health status change across domains; a lower than ideal response rate; the potential for bias in study participation due to sampling method; and under-representation of low-income families.

\section{Conclusion}

The majority of children and adolescents in this study experienced deterioration in their mental health during the initial phase of the implementation of the emergency measures. Further, children with pre-existing MH and/or ASD diagnoses experienced comparable changes in status across domains compared with children/adolescents without preexisting psychiatric diagnoses. The strongest predictor of deterioration of mental health was experiencing increased stress from social isolation. Efforts to maintain or adapt, rather than cancel, children's activities should be encouraged. Public policy, educational systems, and mental health sectors need to balance the risk of infection with the deterioration in child and adolescent mental health noted in this first wave as decisions are made about re-entry to school and recreational activities and other normative activities.

Supplementary Information The online version contains supplementary material available at https://doi.org/10.1007/s00787-021-01744-3.

Acknowledgements The authors would like to gratefully acknowledge Reva Schachter and Avalon Henry for their help with data management; Peter Szatmari for helpful comments on earlier drafts; as well as the families, children, and youth who have participated in this research.

Funding This research was funded by the Canadian Institutes for Health Research; the Ontario Ministry of Health; Centre for Brain and Mental Health, SickKids; Leong Centre for Healthy Children, SickKids; the Miner's Lamp Innovation Fund in Prevention and Early Detection of Severe Mental Illness at the University of Toronto. Additional support for the POND cohort comes from the Ontario Brain Institute.

\section{Compliance with ethical standards}

Conflict of interest The authors declare no commercial conflicts of interest.

\section{References}

1. Brazendale $\mathrm{K}$ et al (2017) Understanding differences between summer vs. school obesogenic behaviors of children: the structured days hypothesis. Int J Behav Nutr Phys Act 14(1):1-14

2. Bridley A, Jordan SS (2012) Child routines moderate daily hassles and children's psychological adjustment. Children's Health Care 41(2):129-144

3. Biddle SJ, Asare M (2011) Physical activity and mental health in children and adolescents: a review of reviews. Br J Sports Med 45(11):886-895

4. Xie X, Xue Q, Zhou Y, Zhu K, Liu Q, Zhang J, Song R (2020) Mental health status among children in home confinement during the coronavirus disease 2019 outbreak in Hubei Province. China. JAMA Pediatrics

5. Zhou S-J, Zhang L-G, Wang L-L, Guo Z-C, Wang J-Q, Chen J-C, Liu M, Chen X, Chen J-X (2020) Prevalence and socio-demographic correlates of psychological health problems in Chinese 
adolescents during the outbreak of COVID-19. Eur Child Adolesc Psychiatry $1-10$

6. Patrick SW, Henkhaus LE, Zickafoose JS, Lovell K, Halvorson A, Loch S, Letterie M, Davis MM (2020) Well-being of parents and children during the COVID-19 pandemic: a national survey. Pediatrics 146(4)

7. Gassman-Pines A, Ananat EO, Fitz-Henley J (2020) COVID-19 and parent-child psychological well-being. Pediatrics 146(4)

8. Ravens-Sieberer U, Kaman A, Erhart M, Devine J, Schlack R, Otto C (2020) Impact of the COVID-19 pandemic on quality of life and mental health in children and adolescents. SSRN 3721508

9. Ezpeleta L et al (2020) Life conditions during COVID-19 lockdown and mental health in Spanish adolescents. Int J Environ Res Public Health 17(19):7327

10. Clemens V, Deschamps P, Fegert JM, Anagnostopoulos D, Bailey S, Doyle M, Eliez S, Hansen AS, Hebebrand J, Hillegers M (2020) Potential effects of "social" distancing measures and school lockdown on child and adolescent mental health. Eur Child Adolesc Psychiatry

11. Maria M (2020) No child is an island: sociability in times of social distancing. Eur Child Adolesc Psychiatry 29(7):901-902

12. Dvorsky MR, Breaux R, Becker SP (2020) Finding ordinary magic in extraordinary times: child and adolescent resilience during the COVID-19 pandemic. Eur Child Adolesc Psychiatry 1-3

13. Bruining H, Bartels M, Polderman TJ, Popma A (2020) COVID19 and child and adolescent psychiatry: an unexpected blessing for part of our population? Eur Child Adolesc Psychiatry 1-2

14. POND Network (2020) Cited 2020 August 20. https://pond-netwo rk.ca/

15. Carsley S, Borkhoff CM, Maguire JL, Birken CS, Khovratovich M, McCrindle B, Macarthur C, Parkin PC (2015) Cohort profile: the applied research group for kids (TARGet Kids!). Int J Epidemiol 776-788

16. Spit for Science (2020) [cited 2020 August 24]. https://lab.resea rch.sickkids.ca/schachar/spit-for-science/

17. Harris PA et al (2019) The REDCap consortium: building an international community of software platform partners. J Biomed Inform 95:103208

18. Harris PA et al (2009) Research electronic data capture (REDCap) - a metadata-driven methodology and workflow process for providing translational research informatics support. $\mathrm{J}$ Biomed Inform 42(2):377-381

19. Hawke LD, Monga S, Korczak D, Hayes E, Relihan J, Darnay K, Cleverley K, Lunsky Y, Szatmari P, Henderson J (2020) Impacts of the COVID-19 pandemic on youth mental health among youth with physical health challenges. Early Interv Psychiatry. https:// doi.org/10.1111/eip.13052

20. Nikolaidis A, Paksarian D, Alexander L, DeRosa J, Dunn J, Nielson DM, Droney I, Kang M, Douka I, Bromet E, Milham MP, Stringaris A, Merikangas KR (2020) The Coronavirus Health and Impact Survey (CRISIS) reveals reproducible correlates of pandemic-related mood states across the Atlantic. medRxiv. $p$ 2020.08.24.20181123. https://doi.org/10.1101/2020.08.24.20181 123

21. Elo A-L, Leppänen A, Jahkola A (2003) Validity of a singleitem measure of stress symptoms. Scand J Work Environ Health 444-451

22. Young Q-R et al (2015) Single-item measures for depression and anxiety: validation of the Screening Tool for Psychological Distress in an inpatient cardiology setting. Eur J Cardiovasc Nurs 14(6):544-551

23. Croft $\mathrm{S}$ et al (2015) Validity of the strengths and difficulties questionnaire in preschool-aged children. Pediatrics 135(5):e1210-e1219

24. Goodman R (1997) The strengths and difficulties questionnaire: a research note. J Child Psychol Psychiatry 38(5):581-586
25. Oliver BR (2015) Unpacking externalising problems: negative parenting associations for conduct problems and irritability. BJPsych Open 1(1):42-47

26. Sukhodolsky DG et al (2016) Behavioral interventions for anger, irritability, and aggression in children and adolescents. J Child Adolesc Psychopharmacol 26(1):58-64

27. Merikangas $\mathrm{K}$ et al (2020) The corona virus Health Impact Survey (CRISIS). https://github.com/nimh-mbdu.CRISIS. Accessed 15 May 2020

28. Lai M-C et al (2020) The CoRonavIruS Health Impact Survey (CRISIS) - adapted for autism and related neurodevelopmental conditions (AFAR) [cited 2020 May 15]. www.crisissurvey.org/ crisis-afar/

29. Team RC (2015) R: a language and environment for statistical computing. R Foundation for Statistical Computing, Vienna

30. Team R (2018) RStudio: integrated development for R. 2018, RStudio, Inc., Boston

31. van Buuren S, Groothuis-Oudshoorn K (2019) mice: multivariate imputation by chained equations. Comprehensive R Archive Network

32. Lewin A et al (2018) Attrition bias related to missing outcome data. Epidemiology 29(1):87-95

33. Rombach I et al (2018) Multiple imputation for patient reported outcome measures in randomised controlled trials: advantages and disadvantages of imputing at the item, subscale or composite score level. BMC Med Res Methodol 18(1):87

34. Madley-Dowd P et al (2019) The proportion of missing data should not be used to guide decisions on multiple imputation. J Clin Epidemiol 110:63-73

35. Patterson J et al (2002) Improving mental health through parenting programmes: block randomised controlled trial. Arch Dis Child 87(6):472-477

36. Bayaga A (2010) Multinomial logistic regression: usage and application in risk analysis. J Appl Quant Methods 5(2)

37. Enhanced Epidemiological Summary (2020) COVID-19 in Ontario-a focus on diversity. 2020 [cited 2020 August 20]. https://www.publichealthontario.ca/-/media/documents/ncov/ epi/2020/06/covid-19-epi-diversity.pdf?la=en

38. Majoko T, Dudu A (2020) Parents' strategies for home educating their children with autism spectrum disorder during the COVID19 period in Zimbabwe. Int J Dev Disabil 1-5

39. Lim $\mathrm{T}$ et al (2020) Autism spectrum disorder and COVID-19: helping caregivers navigate the pandemic. Ann Acad Med Singap 49(6):384-386

40. Jiao WY, Wang LN, Liu J, Fang SF, Jiao FY, Pettoello-Mantovani M, Somekh E (2020) Behavioral and emotional disorders in children during the COVID-19 epidemic. Mosby Inc. 221:264-266. e1. https://doi.org/10.1016/j.jpeds.2020.03.013

41. Pereira-Ávila FMV et al (2020) Public utilization of face masks in Brazil during COVID-19 pandemic outbreak: temporal trend analysis. https://doi.org/10.21203/rs.3.rs-48087/v1

42. Saurabh K, Ranjan S (2020) Compliance and psychological impact of quarantine in children and adolescents due to Covid-19 pandemic. Indian J Pediatr 87(7):532-536. https://doi.org/10.1007/ s12098-020-03347-3

43. Spinelli M et al (2020) Parents' stress and children's psychological problems in families facing the COVID-19 outbreak in Italy. Front Psychol 11:1713

44. Zhang J et al (2020) Acute stress, behavioural symptoms and mood states among school-age children with attention-deficit/ hyperactive disorder during the COVID-19 outbreak. Asian J Psychiatry 51:102077

45. Stress in America (2020) American Psychological Association, USA

46. Parameshwaran K (2020) Universal basic income. AMSA J Glob Health 14(1):42-47 
47. Gibson M, Hearty W, Craig P (2020) The public health effects of interventions similar to basic income: a scoping review. Lancet
Public Health 5(3):e165-e176

\section{Authors and Affiliations}

Katherine Tombeau Cost ${ }^{1}$ [ $\cdot$ Jennifer Crosbie ${ }^{1,2} \cdot$ Evdokia Anagnostou $^{3,4} \cdot$ Catherine S. Birken $^{3,5} \cdot$ Alice Charach $^{1,2}$. Suneeta Monga ${ }^{1,2} \cdot$ Elizabeth Kelley $^{6} \cdot$ Rob Nicolson $^{7} \cdot$ Jonathon L. Maguire ${ }^{3,8} \cdot$ Christie L. Burton $^{1}$. Russell J. Schachar ${ }^{1,2} \cdot$ Paul D. Arnold ${ }^{9,10} \cdot$ Daphne J. Korczak ${ }^{1,2}$

1 Department of Psychiatry, Hospital for Sick Children, Peter Gilgan Centre for Research and Learning, 686 Bay Street, 6th Floor, Toronto, On M5G 0A4, Canada

2 Department of Psychiatry, Faculty of Medicine, University of Toronto, Toronto, Canada

3 Department of Pediatrics, Faculty of Medicine, University of Toronto, Toronto, ON, Canada

4 Holland Bloorview Research Institute, Toronto, ON, Canada

5 Division of Paediatric Medicine, Hospital for Sick Children, Toronto, ON, Canada

6 Department of Psychology, Queens University, Kingston, ON, Canada
7 Department of Psychiatry, University of Western Ontario, London, ON, Canada

8 MAP Centre for Urban Health Solutions, Li Ka Shing Knowledge Institute, Unity Health Toronto, Toronto, Canada

9 Mathison Centre for Mental Health Research and Education, Hotchkiss Brain Institute, University of Calgary, Calgary, AB, Canada

10 Department of Psychiatry, Cumming School of Medicine, University of Calgary, Calgary, AB, Canada 